\title{
cccreative
}

ISSN 2590-9770

The Art of Discrete and Applied Mathematics 3 (2020) \#P2.03

https://doi.org/10.26493/2590-9770.1241.7f8

(Also available at http://adam-journal.eu)

\section{Some remarks on Balaban and sum-Balaban index ${ }^{*}$}

\author{
Martin Knor \\ Slovak University of Technology in Bratislava, Faculty of Civil Engineering, \\ Department of Mathematics, Radlinského 11, 813 68, Bratislava, Slovakia
}

\section{Jozef Komorník}

Faculty of Management, Comenius University, Odbojárov 10, 83104 Bratislava

\section{Riste Škrekovski}

FMF, University of Ljubljana, Jadranska ulica 19, 1000 Ljubljana, Slovenia, and Faculty of Information Studies, Ljubljanska cesta 31a, 8000 Novo mesto, Slovenia, and FAMNIT, University of Primorska, Glagoljaška, 6000 Koper, Slovenia

\section{Aleksandra Tepeh ${ }^{\dagger}$}

Faculty of Information Studies, Ljubljanska cesta 31a, 8000 Novo mesto, Slovenia, and Faculty of Electrical Engineering and Computer Science, University of Maribor, Koroška cesta 46, 2000 Maribor, Slovenia

Received 3 February 2018, accepted 14 March 2019, published online 4 May 2020

\begin{abstract}
In the paper we study maximal values of Balaban and sum-Balaban index, and correct some results appearing in the literature which are only partially correct. Henceforth, we were able to solve a conjecture of M. Aouchiche, G. Caporossi and P. Hansen regarding the comparison of Balaban and Randić index. In addition, we showed that for every $k$ and large enough $n$, the first $k$ graphs of order $n$ with the largest value of Balaban index are trees. We conclude the paper with a result about the accumulation points of sum-Balaban index.
\end{abstract}

Keywords: Topological index, Balaban index, sum-Balaban index, Randić index.

Math. Subj. Class. (2020): 05C12, 05C90

*The authors acknowledge partial support by Slovak research grants VEGA 1/0142/17, VEGA 1/0238/19, APVV-15-0220 and APVV-17-0428, Slovenian research agency ARRS, program no. P1-0383, and National Scholarship Programme of the Slovak Republic SAIA.

$\dagger$ Corresponding author.

E-mail addresses: knor@math.sk (Martin Knor), jozef.komornik@fm.uniba.sk (Jozef Komorník), skrekovski@gmail.com (Riste Škrekovski), aleksandra.tepeh@gmail.com (Aleksandra Tepeh)

()( This work is licensed under https://creativecommons.org/licenses/by/4.0/ 


\section{Introduction}

In this paper we consider simple and connected graphs. For a graph $G$, by $V(G)$ and $E(G)$ we denote the vertex and edge sets of $G$, respectively. Let $n=|V(G)|$ and $m=|E(G)|$. For vertices $u, v \in V(G)$, by $\operatorname{dist}_{G}(u, v)$ (or shortly just $\operatorname{dist}(u, v)$ ) we denote the distance from $u$ to $v$ in $G$, and by $w(u)$ we denote the transmission (or the status) of $u$, defined as $w(u)=\sum_{x \in V(G)} d_{G}(u, x)$.

Balaban index and sum-Balaban index are two of many distance-based topological indices, which are widely used in QSAR/QSPR modeling. Balaban index $J(G)$ of a connected graph $G$, defined as

$$
J(G)=\frac{m}{m-n+2} \sum_{e=u v} \frac{1}{\sqrt{w(u) \cdot w(v)}},
$$

was introduced in early eighties by Balaban [2,3]. Later Balaban et al. [4] (and independently also Deng [9]) proposed a derived measure, namely the sum-Balaban index $\mathrm{SJ}(G)$ for a connected graph $G$ :

$$
\mathrm{SJ}(G)=\frac{m}{m-n+2} \sum_{u v \in E(G)} \frac{1}{\sqrt{w(u)+w(v)}} .
$$

Although sum-Balaban index was introduced just a few years ago, several interesting results have already been published. Regarding extremal values, it was shown by Deng [9] and Xing et al. [23] that for a tree $T$ on $n$ vertices, $n \geq 2$,

$$
\mathrm{SJ}\left(P_{n}\right) \leq \mathrm{SJ}(T) \leq \mathrm{SJ}\left(S_{n}\right)
$$

with left (right, resp.) equality if and only if $T=P_{n}$ ( $T=S_{n}$, resp.), where $P_{n}$ is the path on $n$ vertices and $S_{n}$ is the star on $n$ vertices. In [23] also trees with the second-largest, and third-largest (as well as the second-smallest, and third-smallest) sum-Balaban index among the $n$-vertex trees for $n \geq 6$ were determined. In [15] alternative proof for the above results and further ranking up to seventh maximum sum-Balaban index was presented.

In [26] the authors investigated the maximum value of sum-Balaban index for trees with a given diameter. The extremal graphs which attain the maximum sum-Balaban index among trees with given number of vertices and maximum degree, are determined in [25]. Unicyclic graphs on $n$ vertices with the maximum value of sum-Balaban index were considered in [24], and $n$-vertex bicyclic graphs were studied in [6, 11].

For various upper and lower bounds on general graphs in terms of some other parameters (such as the maximum degree, number of edges, etc.) see [9] and [23], and for recent results on $r$-regular graphs, see [20].

Balaban index is somewhat better explored. We refer an interested reader to $[13,14$, 16, 18] for recent papers, and to [19] for a survey. Despite the fact that Balaban index was introduced much earlier, some of its basic properties, such as the smallest possible value among all $n$-vertex graphs, are still unknown.

Balaban index was originally named as the "average distance-sum connectivity index". It is based on a Randić type formula, today called the Randic index [21], and known also as the connectivity index $R(G)$ of a graph $G$, defined by

$$
R(G)=\sum_{u v \in E(G)} \frac{1}{\sqrt{\operatorname{deg}(u) \cdot \operatorname{deg}(v)}},
$$


where $\operatorname{deg}(u)(\operatorname{deg}(v)$, resp.) denotes the degree of $u$ ( $v$, resp.) in $G$. Note that in the definition of Balaban index, vertex degrees are replaced by transmissions.

With this paper we would like to contribute to better understanding of maximal values of both indices, and correct erroneous statements that appeared in the literature regarding some of these values (see Section 2). In addition, having correct results, we were able to show in Section 3 that a conjecture from [1] regarding the comparison of Balaban and Randić index holds. We conclude the paper with a result about the accumulation points of sum-Balaban index. The result is based on a proof of an upper bound for the minimum value of $\mathrm{SJ}(G)$.

\section{Maximum values}

The cyclomatic number $\mu$ of $G$, which is the minimum number of edges that must be removed from $G$ in order to transform it to an acyclic graph, equals $m-n+1$. Note that the denominator $m-n+2$ in the definition of Balaban and sum-Balaban index can be expressed as $\mu+1$. In this section we determine maximal values for both indices for graphs which contain at least one cycle.

Theorem 2.1. Let $G$ be a connected graph on $n$ vertices with $\mu \geq 1$. Then:

(1) $J(G)$ is maximum if and only of $G$ is the complete graph $K_{n}$;

(2) $\mathrm{SJ}(G)$ is maximum if and only of $G$ is the complete graph $K_{n}$.

Proof. Since $G$ is a connected graph with $\mu \geq 1$, we have $n \geq 3$ and $m \in\left[n,\left(\begin{array}{c}n \\ 2\end{array}\right)\right]$. For every $u \in V(G)$, we have $w(u) \geq n-1$, which implies

$$
J(G) \leq \frac{m^{2}}{(m-n+2)(n-1)},
$$

with equality if and only if $G=K_{n}$.

Let $G$ be a graph on $n$ vertices which is not complete. In order to prove that $J(G)<$ $J\left(K_{n}\right)=\frac{n^{2}(n-1)}{2\left(n^{2}-3 n+4\right)}$, one needs to check that for every $n \geq 3$ and $m \in\left[n,\left(\begin{array}{c}n \\ 2\end{array}\right)\right]$ we have

$$
\frac{m^{2}}{(m-n+2)(n-1)} \leq \frac{n^{2}(n-1)}{2\left(n^{2}-3 n+4\right)},
$$

or equivalently

$$
-2 m^{2}\left(n^{2}-3 n+4\right)+n^{2}(n-1)^{2}(m-n+2) \geq 0 .
$$

Let $f(m)$ be the left-hand side of (2.3), i.e., $f(m)=-2 m^{2}\left(n^{2}-3 n+4\right)+n^{2}(n-$ $1)^{2}(m-n+2)$. Then $f$ is quadratic in $m$ with a negative leading coefficient. Hence, $f(m)$ is concave. Since

$$
f(n)=n^{2}(2 n-6) \geq 0 \quad \text { and } \quad f\left(\frac{n(n-1)}{2}\right)=0,
$$

we conclude that $f(m) \geq 0$ for every $m \in\left[n,\left(\begin{array}{l}n \\ 2\end{array}\right)\right]$. Hence (2.3) is true, which completes the proof for Balaban index.

To prove the statement for sum-Balaban index, observe that since $w(u) \geq n-1$, we have

$$
\mathrm{SJ}(G) \leq \frac{m^{2}}{(m-n+2) \sqrt{2 n-2}},
$$


with equality if and only if $G=K_{n}$.

Let $G$ be a graph on $n$ vertices which is not complete. In order to prove that $\mathrm{SJ}(G)<$ $\mathrm{SJ}\left(K_{n}\right)=\frac{n^{2}(n-1)^{2}}{2\left(n^{2}-3 n+4\right) \sqrt{2 n-2}}$, one needs to check that for every $n \geq 3$ and $m \in\left[n,\left(\begin{array}{l}n \\ 2\end{array}\right)\right]$ we have

$$
\frac{m^{2}}{(m-n+2) \sqrt{2 n-2}} \leq \frac{n^{2}(n-1)^{2}}{2\left(n^{2}-3 n+4\right) \sqrt{2 n-2}} .
$$

Since the above inequality is equivalent to (2.2), the proof is complete.

Sun [22], and Dong and Guo [10], independently studied Balaban index of trees with given number of vertices. Their results hold, however Deng [8] corrected mistakes in their proofs of the statement that for a tree $T$ on $n \geq 2$ vertices it holds

$$
J\left(P_{n}\right) \leq J(T) \leq J\left(S_{n}\right)
$$

with left (right, resp.) equality if and only if $T=P_{n}$ ( $T=S_{n}$, resp.).

In [10] the authors also state that for a connected graph $G$ with $n$ vertices

$$
J(G) \leq J\left(S_{n}\right)=\sqrt{\frac{(n-1)^{3}}{2 n-3}},
$$

with equality if and only if $G=S_{n}$. It was brought to our attention that two years later (seemingly unaware of the paper by Dong and Guo), Aouchiche et al. [1] posed the conjecture, which we can state here as a theorem.

Theorem 2.2. For any connected graph $G$ on $n \geq 2$ vertices, we have

$$
J(G) \leq \begin{cases}J\left(K_{n}\right), & \text { if } n \leq 7 \\ J\left(S_{n}\right), & \text { if } n \geq 8\end{cases}
$$

In their proof, Dong and Guo use the assumption that $n \geq 9$ and neglect smaller cases. By Theorem 2.1 and (2.5), to complete the proof of Theorem 2.2 it suffices to compare

$$
J\left(S_{n}\right)=\sqrt{\frac{(n-1)^{3}}{2 n-3}} \quad \text { and } \quad J\left(K_{n}\right)=\frac{n^{2}(n-1)}{2\left(n^{2}-3 n+4\right)}
$$

for $n \in[3,8]$. It turns out that $J\left(S_{n}\right)<J\left(K_{n}\right)$ if $n \in[3,7]$, while $J\left(S_{8}\right)>J\left(K_{8}\right)$.

For sum-Balaban index we have an analogous result.

Theorem 2.3. For any connected graph $G$ on $n \geq 2$ vertices, we have

$$
\mathrm{SJ}(G) \leq \begin{cases}\mathrm{SJ}\left(K_{n}\right), & \text { if } n \leq 5 \\ \mathrm{SJ}\left(S_{n}\right), & \text { if } n \geq 6 .\end{cases}
$$

Proof. By Theorem 2.1 and (1.1), it suffices to compare

$$
\mathrm{SJ}\left(S_{n}\right)=\frac{(n-1)^{2}}{\sqrt{3 n-4}} \quad \text { and } \quad \operatorname{SJ}\left(K_{n}\right)=\frac{n^{2}(n-1)^{2}}{2\left(n^{2}-3 n+4\right) \sqrt{2 n-2}} .
$$

By a computer one can check that

$$
f(x)=\frac{1}{\sqrt{3 x-4}}-\frac{x^{2}}{2\left(x^{2}-3 x+4\right) \sqrt{2 x-2}}
$$

has only two roots on $[2, \infty)$, namely 2 (in which case $S_{2}=K_{2}$ ) and 5.5543. Since $\mathrm{SJ}\left(S_{5}\right)-\mathrm{SJ}\left(K_{5}\right)<0$ and $\mathrm{SJ}\left(S_{6}\right)-\mathrm{SJ}\left(K_{6}\right)>0$, we conclude the result. 
In [10] the authors state a problem of characterizing graphs with the maximum (the minimum) Balaban index among $k$-connected ( $k$-edge-connected) graphs on $n$ vertices. Although the case of the minumum Balaban index may be hard to solve, Theorems 2.1 and 2.2 yield the following corollary.

Corollary 2.4. Let $G$ be a graph with the maximum value of Balaban index in the class of $k$-connected ( $k$-edge-connected) graphs of order $n$. Then we have:

(1) if $k=1$ and, $n=2$ or $n \geq 8$, then $G$ is the star $S_{n}$;

(2) if $k=1$ and $n \leq 7$, or $k \geq 2$, then $G$ is the complete graph $K_{n}$.

Analogously, by Theorems 2.1 and 2.3 we have:

Corollary 2.5. Let $G$ be a graph with the maximum value of sum-Balaban index in the class of $k$-connected ( $k$-edge-connected) graphs of order $n$. Then we have:

(1) if $k=1$ and, $n=2$ or $n \geq 6$, then $G$ is the star $S_{n}$;

(2) if $k=1$ and $n \leq 5$, or $k \geq 2$, then $G$ is the complete graph $K_{n}$.

By the proof of Theorem 2.1, $J\left(K_{n}\right) \sim \frac{n}{2}$ while for every tree $T$ we have $J(T) \sim \frac{n^{2}}{\bar{w}}$, where $\bar{w}$ is the harmonic mean of $\{\sqrt{w(u) \cdot w(v)} ; u v \in E(G)\}$, i.e.

$$
\bar{w}=\frac{m}{\sum_{u v \in E(G)} \frac{1}{\sqrt{w(u) \cdot w(v)}},}
$$

(note that $\left.J(G)=\frac{m^{2}}{(m-n+2) \bar{w}}\right)$. This means that, roughly speaking, if $\bar{w}<2 n$, then $J(T)>J\left(K_{n}\right)$.

Denote by $D_{a, b}^{*}$ a tree on $a+b$ vertices, one of which has degree $a$, another has degree $b$, and all the other vertices have degree 1 . Then $D_{a, b}^{*}$ is the double star. Observe that if a tree has diameter 2 , then it is a star, while if it has diameter 3 , it is a double star.

Theorem 2.6. Let $a$ and $b$ be positive integers such that $a, b \geq 2, a+b=n$ and $n \geq 9$. Then $J\left(D_{a, b}^{*}\right)>J\left(K_{n}\right)$.

Proof. Consider the double star $D_{a, b}^{*}$. Let $u_{2}$ and $u_{3}$ be the vertices of degree $a$ and $b$, respectively. Moreover, let $u_{1}\left(u_{4}\right.$, resp.) be a pendant vertex adjacent to $u_{2}\left(u_{3}\right.$, resp.). Since $b=n-a$, we have

$$
\begin{aligned}
& w\left(u_{1}\right)=1+2(a-1)+3(b-1)=3 n-a-4, \\
& w\left(u_{2}\right)=a+2(b-1)=2 n-a-2, \\
& w\left(u_{3}\right)=b+2(a-1)=n+a-2, \\
& w\left(u_{4}\right)=1+2(b-1)+3(a-1)=2 n+a-4 .
\end{aligned}
$$

Hence,

$$
\begin{aligned}
f(a)= & \sum_{u v \in E(G)} \frac{1}{\sqrt{w(u) \cdot w(v)}} \\
= & \frac{a-1}{\sqrt{(3 n-a-4)(2 n-a-2)}}+\frac{1}{\sqrt{(2 n-a-2)(n+a-2)}} \\
& \quad+\frac{n-a-1}{\sqrt{(n+a-2)(2 n+a-4)}},
\end{aligned}
$$


and $J\left(D_{a, b}^{*}\right)=(n-1) f(a)$.

In [8], see the text before Theorem 4, it is proved that $f^{\prime \prime}(x)>0$, which means that $f(x)$ is a convex function. Since $f(a)=f(n-a), 2 \leq a \leq n-2$, we have

$$
J\left(D_{2, n-2}^{*}\right)>J\left(D_{3, n-3}^{*}\right)>\cdots>J\left(D_{\lfloor n / 2\rfloor,\lceil n / 2\rceil}^{*}\right) \geq(n-1) f(n / 2) .
$$

If $n \geq 70$, then $(n-1) f(n / 2)>\frac{n^{2}(n-1)^{2}}{2 n(n-3)+8} \cdot \frac{1}{n-1}=J\left(K_{n}\right)$, which implies that $J\left(D_{a, b}^{*}\right)>$ $J\left(K_{n}\right)$ in this case. The cases when $n<70$ were checked using a computer software.

Theorem 2.6 implies the following.

Corollary 2.7. For every $k$ there exists $n_{0}$ such that for every $n \geq n_{0}$ the first $k$ graphs of order $n$ with the biggest value of Balaban index are trees.

Analogous result can be proved for sum-Balaban index:

Theorem 2.8. Let $a$ and $b$ be positive integers such that $a, b \geq 2, a+b=n$ and $n \geq 8$. Then $\operatorname{SJ}\left(D_{a, b}^{*}\right)>\operatorname{SJ}\left(K_{n}\right)$.

Proof. Using the values $w$ from the proof of Theorem 2.6 we get

$$
f(a)=\sum_{u v \in E(G)} \frac{1}{\sqrt{w(u)+w(v)}}=\frac{a-1}{\sqrt{5 n-2 a-6}}+\frac{1}{\sqrt{3 n-4}}+\frac{n-a-1}{\sqrt{3 n+2 a-6}},
$$

and $\operatorname{SJ}\left(D_{a, b}^{*}\right)=(n-1) f(a)$. In [23, Lemma 3.2] it is proved that

$$
\mathrm{SJ}\left(D_{2, n-2}^{*}\right)>\operatorname{SJ}\left(D_{3, n-3}^{*}\right)>\cdots>\operatorname{SJ}\left(D_{\lfloor n / 2\rfloor,\lceil n / 2\rceil}^{*}\right) \geq(n-1) f(n / 2) .
$$

Since $(n-1) f(n / 2)>\frac{n^{2}(n-1)^{2}}{2\left(n^{2}-3 n+4\right) \sqrt{2 n-2}}=\operatorname{SJ}\left(K_{n}\right)$ if $n \geq 8$, we have $\operatorname{SJ}\left(D_{a, b}^{*}\right)>$ $\mathrm{SJ}\left(K_{n}\right)$.

Corollary 2.9. For every $k$ there exists $n_{0}$ such that for every $n \geq n_{0}$ the first $k$ graphs of order $n$ with the biggest value of sum-Balaban index are trees.

\section{Comparison with Randić index}

In the class of trees, the star $S_{n}$ maximizes the Balaban index [8, 10, 22] and minimizes the Randić index [5]. Hence, for every tree $T$ we have

$$
\frac{J(T)}{R(T)} \leq \frac{n-1}{\sqrt{2 n-3}}
$$

with equality if and only if $T$ is the star $S_{n}$. This observation was pointed out by Aouchiche et al. [1], who proposed to study an extension of this bound to the class of all connected graphs. Based on their computer experiments for $n \geq 5$ they proposed the conjecture, which turns out to be true (see Theorem 3.1). Namely, by Theorem 2.2, for $n \geq 8$, the star $S_{n}$ is the graph that maximizes the Balaban index over the class of $n$-vertex connected graphs, and over this class of graphs $S_{n}$ also minimizes the Randic index [5, 27]. Using a computer program we have checked that the result holds also for $n \in\{5,6,7\}$, however, for $n \in\{3,4\}$, the quotient $\frac{J(G)}{R(G)}$ attains its maximal value for the complete graph $K_{n}$. Thus we can state the following. 
Theorem 3.1. For any connected graph $G$ on $n \geq 2$ vertices, we have

$$
\frac{J(G)}{R(G)} \leq \begin{cases}\frac{n^{2}-n}{n^{2}-3 n+4}, & \text { if } n \leq 4 \\ \frac{n-1}{\sqrt{2 n-3}}, & \text { if } n \geq 5\end{cases}
$$

with equality if and only if $G=K_{n}$ for $n \leq 4$, and for $n \geq 5$ equality holds precisely for $G=S_{n}$.

Note that a similar observation can be done for the class of $n$-vertex connected unicyclic graphs. For this class Gao and Lu [12] proved that $S_{n}^{+}$(i.e., the graph obtained from the star $S_{n}$ by adding an edge between two nonadjacent vertices) has the minimum Randic index, but on the other hand it has the maximum Balaban index [7, 24]. In other words,

$$
J(G) \leq J\left(S_{n}^{+}\right)=\frac{n}{2}\left(\frac{1}{2 n-4}+\frac{2}{\sqrt{(2 n-4)(n-1)}}+\frac{n-3}{\sqrt{(2 n-3)(n-1)}}\right),
$$

and

$$
R(G) \geq R\left(S_{n}^{+}\right)=\frac{n-3}{\sqrt{n-1}}+\frac{2}{\sqrt{2(n-1)}}+\frac{1}{2},
$$

for any connected unicyclic graph on at least 4 vertices. Thus we obtain the following result.

Theorem 3.2. For any connected unicyclic graph $G$ on $n \geq 4$ vertices, we have

$$
\frac{J(G)}{R(G)} \leq \frac{J\left(S_{n}^{+}\right)}{R\left(S_{n}^{+}\right)}
$$

with equality if and only if $G=S_{n}^{+}$.

\section{Accumulation points of sum-Balaban index}

In [17] it is shown that for every nonnegative real number $r$ there exists a sequence of graphs $\left\{G_{r, i}\right\}_{i=1}^{\infty}$ such that the number of vertices of $G_{r, i}$ tends to infinity as $i \rightarrow \infty$ and $\lim _{i \rightarrow \infty} J\left(G_{r, i}\right)=r$. Here we prove an analogous result for sum-Balaban index.

Let $K_{a}$ and $K_{a}^{\prime}$ be two disjoint complete graphs on $a$ vertices and let $P_{b}$ be a path on $b$ vertices. The balanced dumbbell graph $D_{a, b}$ is obtained from $K_{a} \cup P_{b} \cup K_{a}^{\prime}$ by joining all vertices of $K_{a}$ to one end-vertex of $P_{b}$ and all vertices of $K_{a}^{\prime}$ to the other end-vertex of $P_{b}$. Thus, $D_{a, b}$ has $2 a+b$ vertices. See Figure 1 for $D_{5,5}$.

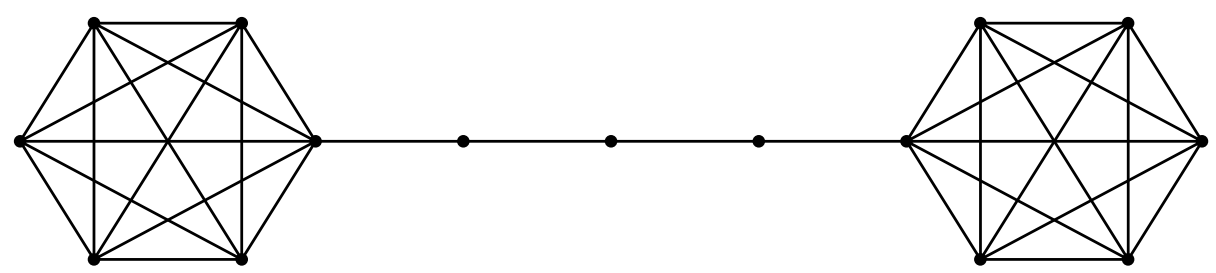

Figure 1: The graph $D_{5,5}$.

Denote $Q=\sqrt{2} \ln (1+\sqrt{2})$. Observe that $Q \doteq 1.24650$ and $1+Q+2 \sqrt{Q} \doteq 4.47934$. We have the following statement. 
Theorem 4.1. Let $r \geq 1+Q+2 \sqrt{Q}$. Further, let $\left\{D_{a_{i}, b_{i}}\right\}_{i=1}^{\infty}$ be a sequence of balanced dumbbell graphs on $n_{i}=2 a_{i}+b_{i}$ vertices such that $n_{i} \rightarrow \infty$ and

$$
\lim _{i \rightarrow \infty} \frac{a_{i}}{\sqrt{n_{i}}}=\frac{1}{\sqrt{2}} \sqrt{r-1-Q+\sqrt{(r-1-Q)^{2}-4 Q}} .
$$

Then $\lim _{i \rightarrow \infty} \operatorname{SJ}\left(D_{a_{i}, b_{i}}\right)=r$.

Proof. First observe that if $r \geq 1+Q+2 \sqrt{Q}$ then $(r-1-Q)^{2}-4 Q \geq 0$, and so $(1 / \sqrt{2}) \sqrt{r-1+Q+\sqrt{(r-1-Q)^{2}-4 Q}}$ is a real number.

In [14, Equation (9)] it is proved that if $a \sim c \sqrt{n}$ for a (real) constant $c$, then for a balanced dumbbell graph $D_{a, b}$ on $n$ vertices it holds

$$
\mathrm{SJ}\left(D_{a, b}\right) \sim c^{2}+1+Q+\frac{Q}{c^{2}}
$$

Hence, for $c=\frac{1}{\sqrt{2}} \sqrt{r-1-Q+\sqrt{(r-1-Q)^{2}-4 Q}}$ we get

$$
\begin{aligned}
\operatorname{SJ}\left(D_{a, b}\right) & \sim \frac{1}{2}\left(r-1-Q+\sqrt{(r-1-Q)^{2}-4 Q}\right)+1+Q \\
& +\frac{2 Q}{r-1-Q+\sqrt{(r-1-Q)^{2}-4 Q}} \\
= & \frac{1}{2}\left(r+\sqrt{(r-1-Q)^{2}-4 Q}+1+Q\right. \\
+\frac{4 Q}{r+\sqrt{(r-1-Q)^{2}-4 Q}-1-Q} & \\
= & \frac{1}{2} \cdot \frac{2 r^{2}+2 r \sqrt{(r-1-Q)^{2}-4 Q}-2 r-2 r Q}{r+\sqrt{(r-1-Q)^{2}-4 Q}-1-Q}=r .
\end{aligned}
$$

Although we have a conjecture that for graphs $G$ on large number of vertices

$$
\mathrm{SJ}(G) \geq 1+Q+2 \sqrt{Q}
$$

(see Corollary 8 and Conjecture 9 in [14]), it is proved only that

$$
\mathrm{SJ}(G) \geq 4+o(1)
$$

(see Theorem 2 in [14]). Hence, if our conjecture is false, then the problem of accumulation points of sum-Balaban index for values in interval $[4,4.47934)$ remains open.

\section{ORCID iDs}

Riste Škrekovski (D) https://orcid.org/0000-0001-6851-3214 Aleksandra Tepeh (D) https://orcid.org/0000-0002-2321-6766

\section{References}

[1] M. Aouchiche, G. Caporossi and P. Hansen, Refutations, results and conjectures about the Balaban index, Internat. J. Chem. Model. 5 (2013), 189-202. 
[2] A. T. Balaban, Highly discriminating distance-based topological index, Chem. Phys. Lett. 89 (1982), 399-404, doi:10.1016/0009-2614(82)80009-2.

[3] A. T. Balaban, Topological indices based on topological distances in molecular graphs, Pure Appl. Chem. 55 (1983), 199-206, doi:10.1351/pac198855020199.

[4] A. T. Balaban, P. V. Khadikar and S. Aziz, Comparison of topological indices based on iterated 'sum' versus 'product' operations, Iranian J. Math. Chem. 1 (2010), 43-67, doi:10.22052/ijmc. 2010.5134.

[5] B. Bollobás and P. Erdős, Graphs of extremal weights, Ars Combin. 50 (1998), 225-233.

[6] Z. Chen, M. Dehmer, Y. Shi and H. Yang, Sharp upper bounds for the Balaban index of bicyclic graphs, MATCH Commun. Math. Comput. Chem. 75 (2016), 105-128, http://match.pmf.kg.ac.rs/electronic_versions/Match75/n1/ match75n1_105-128.pdf.

[7] B. Deng and A. Chang, Maximal Balaban index of graphs, MATCH Commun. Math. Comput. Chem. 70 (2013), 259-286, http://match.pmf.kg.ac.rs/electronic_ versions/Match70/n1/match70n1_259-286.pdf.

[8] H. Deng, On the Balaban index of trees, MATCH Commun. Math. Comput. Chem. 66 (2011), 253-260, http://match.pmf.kg.ac.rs/electronic_versions/ Match66/n1/match66n1_253-260.pdf.

[9] H. Deng, On the sum-Balaban index, MATCH Commun. Math. Comput. Chem. 66 (2011), 273-284, http://match.pmf.kg.ac.rs/electronic_versions/ Match66/n1/match66n1_273-284.pdf.

[10] H. Dong and X. Guo, Character of trees with extreme Balaban index, MATCH Commun. Math. Comput. Chem. 66 (2011), 261-272, http://match.pmf.kg.ac.rs/electronic_ versions/Match66/n1/match66n1_261-272.pdf.

[11] W. Fang, Y. Gao, Y. Shao, W. Gao, G. Jing and Z. Li, Maximum Balaban index and sum-Balaban index of bicyclic graphs, MATCH Commun. Math. Comput. Chem. 75 (2016), 129-156, http://match.pmf.kg.ac.rs/electronic_versions/ Match75/n1/match75n1_129-156.pdf.

[12] J. Gao and M. Lu, On the Randić index of unicyclic graphs, MATCH Commun. Math. Comput. Chem. 53 (2005), 377-384, http://match.pmf.kg.ac.rs/electronic_ versions/Match53/n2/match53n2_377-384.pdf.

[13] M. Knor, J. Kranjc, R. Škrekovski and A. Tepeh, A search for the minimum value of Balaban index, Appl. Math. Comput. 286 (2016), 301-310, doi:10.1016/j.amc.2016.04.023.

[14] M. Knor, J. Kranjc, R. Škrekovski and A. Tepeh, On the minimum value of sum-Balaban index, Appl. Math. Comput. 303 (2017), 203-210, doi:10.1016/j.amc.2017.01.041.

[15] M. Knor, R. Škrekovski and A. Tepeh, Trees with large sum-Balaban index, submitted.

[16] M. Knor, R. Škrekovski and A. Tepeh, Balaban index of cubic graphs, MATCH Commun. Math. Comput. Chem. 73 (2015), 519-528, http://match.pmf.kg.ac.rs/electronic_ versions/Match73/n2/match73n2_519-528.pdf.

[17] M. Knor, R. Škrekovski and A. Tepeh, A note on accumulation points of Balaban index, MATCH Commun. Math. Comput. Chem. 78 (2017), 163-168, http: / match.pmf.kg . ac.rs/electronic_versions/Match78/n1/match78n1_163-168.pdf.

[18] M. Knor, R. Škrekovski and A. Tepeh, Convexity result and trees with large Balaban index, Appl. Math. Nonlinear Sci. 3 (2018), 433-445, doi:10.21042/amns.2018.2.00034. 
[19] M. Knor, R. Škrekovski and A. Tepeh, Mathematical aspects of Balaban index, MATCH Commun. Math. Comput. Chem. 79 (2018), 685-716, http: / /match.pmf.kg.ac.rs/ electronic_versions/Match79/n3/match79n3_685-716.pdf.

[20] H. Lei and H. Yang, Bounds for the sum-Balaban index and (revised) Szeged index of regular graphs, Appl. Math. Comput. 268 (2015), 1259-1266, doi:10.1016/j.amc.2015.07.021.

[21] M. Randić, On characterization of molecular branching, J. Am. Chem. Soc. 97 (1975), 66096615, doi:10.1021/ja00856a001.

[22] L. Sun, Bounds on the Balaban index of trees, MATCH Commun. Math. Comput. Chem. 63 (2010), 813-818, http://match.pmf.kg.ac.rs/electronic_versions/ Match63/n3/match63n3_813-818.pdf.

[23] R. Xing, B. Zhou and A. Graovac, On sum-Balaban index, Ars Combin. 104 (2012), 211-223.

[24] L. You and X. Dong, The maximum Balaban index (sum-Balaban index) of unicyclic graphs, J. Math. Res. Appl. 34 (2014), 392-402, doi:10.3770/j.issn:2095-2651.2014.04.002.

[25] L. You and H. Han, The maximum sum-Balaban index of tree graph with given vertices and maximum degree, Adv. Appl. Math. (Chinese) 2 (2013), 147-151, doi:10.12677/aam.2013. 24019.

[26] L. You and H. Han, The maximum sum-Balaban index of trees with given diameter, Ars Combin. 112 (2013), 115-128.

[27] P. Yu, An upper bound on the Randić of trees, J. Math. Study (Chinese) 31 (1998), 225-230, http://en.cnki.com.cn/Article_en/CJFDTotal-SSYJ199802021.htm. 